\title{
Building Large Microkinetic Models With First-Principles' Accuracy at Reduced Computational Cost
}

\author{
Jonathan E. Sutton and Dionisios G. Vlachos* \\ Catalysis Center for Energy Innovation and Center for Catalytic Science and Technology \\ Department of Chemical and Biomolecular Engineering \\ University of Delaware, Newark, DE 19716 \\ *Corresponding author: e-mail: vlachos@udel.edu; tel. 302-831-2830.
}

\begin{abstract}
We present a systematic hierarchical multiscale framework for parameterization of large microkinetic models that delivers first-principles' accuracy at significantly reduced computational cost. The framework leverages recently introduced first-principles-based semi-empirical methods (FPSEM), such as group additivity and Brønsted-Evans-Polanyi (BEP) relations, for surface reactions, local sensitivity analysis, and a heuristic classification of the order of corrections to produce a hierarchy or family of models of improved accuracy. We demonstrate this approach to the moderate size ethanol steam reforming mechanism on Pt, consisting of 67 species (14 gas, 53 surface) and 160 reversible elementarylike reactions, for which the 'exact' density functional theory (DFT)-based model is available. We find that the majority of refined parameters are surface species free energies and lateral interactions, underscoring the importance of thermodynamics in kinetic mechanisms.
\end{abstract}

Keywords: microkinetic modeling, multiscale modeling, semi-empirical methods, hierarchical modeling, ethanol, reforming. 


\section{Introduction}

Microkinetic modeling(Dumesic et al., 1993) has greatly increased in popularity over the last two decades. Models proposed to date have mainly only considered $C_{2}$ species or smaller.(Salciccioli et al., 2011b) Nevertheless, larger molecules are of practical interest. For example, the hydrolysis of actual biomass (e.g., cellulose and hemi-cellulose) results in $C_{5}$ and $C_{6}$ sugars, and cracking of large hydrocarbons or oligomerization of small ones toward larger chemicals or fuels entails reactants and/or products of large molecular weight. For such systems, the number of parameters to be estimated using first principles methods is beyond current computational resources.

As an illustration, Figure 1Error! Reference source not found. presents the number of intermediates and reactions enumerated in catalytic cracking mechanisms of sugar alcohols with up to six $\mathrm{C}$ atoms, and Table 1Error! Reference source not found. shows the estimated CPU time required for a wholly DFTbased model of ethanol steam reforming. This mechanism has 67 species and 160 reactions, similar to the ethylene glycol ( $\mathrm{C}$ number of 2 ) mechanism in Figure 1Error! Reference source not found.. It is immediately apparent that even relatively simple molecules result in rather large mechanisms, and the mechanism size increases rapidly with increasing $C$ number (note that the enumeration presented in Figure 1Error! Reference source not found. neglects stereoisomers, so the number of possible parameters is actually larger). This implies that using only high level computational techniques (e.g., density functional theory (DFT)) for estimating model parameters becomes impractical for all but the smallest molecules. An exception to this generalization entails small reaction mechanisms, such as the glucose isomerization to fructose, the dehydration of fructose, etc. that encompass a handful of elementary reaction steps under the assumption that cracking (such as retro-aldol reactions) is insignificant.

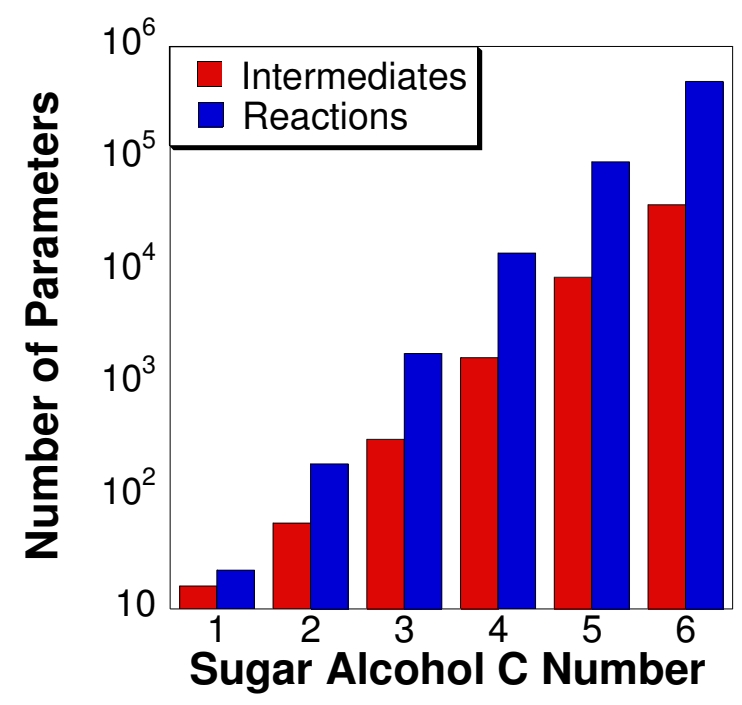

Figure 1. Number of intermediates and reactions as a function of $\mathrm{C}$ number for various sugar alcohols $\mathrm{C}_{x} \mathrm{O}_{x} \mathrm{H}_{(2 x+2)}$. 
Table 1. Summary of estimated DFT computational times for model parameters and total computational time for full DFT-based model. Number of calculations excludes parameters taken from literature ( 6 species and 12 reactions) and non-activated adsorption reactions (11 reactions).

\begin{tabular}{lrrr} 
Parameter Type & CPU-hr/Calculation & Number of Calculations & Total CPU-hr \\
\hline Species Energies at 0 K & 250 & 46 & 11500 \\
Species Free Energies $^{*}$ & 250 & 46 & 11500 \\
Activation Energies at 0 K $^{*}$ & 500 & 137 & 68500 \\
Activation Free Energies $^{*}$ & 500 & 7 & 3500 \\
\hline Total & & & 95000
\end{tabular}

* Represents the computational cost for the vibrational frequency calculations.

Several possible solutions to this challenge have been proposed including Single-Event Microkinetic models (see, e.g., (Thybaut and Marin, 2013) and the references therein) and hierarchical refinement.(Mhadeshwar and Vlachos, 2005; Salciccioli et al., 2011b) In Single-Event Microkinetic modeling, a full microkinetic model is postulated and then reaction family-based constraints are defined to reduce the number of independent parameters. The remaining independent parameters are then regressed from experimental data. In the hierarchical refinement methodology, inexpensive methods are employed to rapidly screen potentially important kinetic parameters (species free energies and reaction activation energies). Such a method has been used to guide DFT calculations for mapping out plausible pathways for glycerol decomposition.(Chen et al., 2011) For quantitative modeling, one can first formulate a complete microkinetic model with parameters estimated using inexpensive methods, identify the most influential parameters, and then refine these influential parameters using either computational methods or experimental data.(Mhadeshwar and Vlachos, 2005) In principle, the concepts of hierarchical refinement are also applicable to Single-Event Microkinetic models.

Previous studies laid the foundation for hierarchical refinement. Mhadeshwar and Vlachos (Mhadeshwar and Vlachos, 2005) successfully demonstrated the basic principle of using semi-empirical methods (in their case via bond order conservation) as a base of parameter estimation of microkinetic models to be refined with more accurate parameters, and Chen et al.(Chen et al., 2011) showed that more recent first-principles based semi-empirical methods (FPSEM), such as group additivity and Brønsted-Evans-Polanyi (BEP) correlations, can be used to map out reaction networks with reduced computational cost. While powerful as an approach, hierarchical refinement of microkinetic models parameterized with recent FPSEM parameters has not been studied in a systematic way and a systematic quantitative convergence test has never been demonstrated before. Our purpose is to incorporate the recent FPSEM into the existing hierarchical multiscale modeling framework and to evaluate its performance.

We carry out these goals by (1) reviewing the computational methods for estimating parameters and solving the resulting microkinetic model, (2) outlining the formal algorithm for carrying out the hierarchical refinement and classifying the relative accuracies of different parameter estimation methods, and (3) applying the proposed method to the development and refinement of a microkinetic model of ethanol steam reforming on Pt. In this case study, we evaluate the convergence of the FPSEMbased microkinetic model to the DFT-based model as parameters are sequentially refined and we estimate the computational cost for the refinement. 


\section{Computational Methods}

\section{Kinetic Parameter Estimation}

For this work, we develop two microkinetic models, based primarily on density functional theory (DFT) and FPSEM calculations, and referred to as the DFT and FPSEM models, respectively. Thermochemical parameters for the gas-phase species in both models were taken from polynomial fits to experimental data (Goos et al., 2014) and are considered to be exact. Both models also incorporated a full DFT-based water-gas shift mechanism taken from the literature (note this mechanism includes the free energies for many of the important small species, such as $\mathrm{CO}^{*}$ and $\mathrm{H}^{*}$ ).(Stamatakis et al., 2011) Thermodynamic discrepancies arising from the use of experimental energies for gas phase species rather than DFT values were reconciled via the method of Blaylock et al.(Blaylock et al., 2009) (values from our group additivity scheme are referenced to the same set of DFT energies).

The surface species energies and reaction activation energies for the DFT model were taken from published DFT results employing the PBE functional on 2x2 and 3×3 unit cells. (Chen and Vlachos, 2010; Chen and Vlachos, 2012; Sutton et al., 2013) One can view this as being the 'exact' model. The second employs adsorbate free energies and reaction activation energies from FPSEM as initial estimates. In both models, all parameters for the surface species and reactions are subject to some inherent uncertainty (e.g., from imperfect DFT exchange/correlation models, the pressure and materials gaps between model and real surfaces, regression errors, etc.) and should be viewed only as estimates, rather than exact values. In the FPSEM model, the species free energies were estimated with group additivity. The activation energies were estimated using BEP correlations, which were divided into five homologous series ( $\mathrm{C}-\mathrm{H}, \mathrm{O}-\mathrm{H}, \mathrm{C}-\mathrm{C}, \mathrm{C}-\mathrm{OH}$, and $\mathrm{C}-\mathrm{O})$, in line with our previous findings,(Sutton and Vlachos, 2012) excluding the values for ethanol dehydroxylation and $\mathrm{CCOH}^{*}$ dehydrogenation, as these were found to deviate significantly from the trends of the remaining points. This model is then refined as described below to generate a hierarchy (a family) of refined microkinetic models.

The species free energies and activation energies were treated as the independent model parameters and then used on-the-fly to calculate the forward and reverse rate constants in a thermodynamically consistent manner. The forward rate constant is estimated using transition state theory

$$
k_{f}=\frac{k_{B} T}{h} \exp \left(\frac{-E_{A}}{R T}\right)
$$

In this formulation, the activation energy is actually a free energy (i.e., incorporating the entropic contributions normally expressed as a ratio of partition functions). For most reactions in both models, entropic contributions to most activation energies are neglected as this assumption has previously been shown to provide reasonable estimates for reduced computational effort.(Sutton et al., 2013) Entropic corrections are applied to all species energies and to selected activation energies as identified during the hierarchical refinement procedure (note we carried out a hierarchical refinement procedure in our previous work on the DFT model to identify which reactions required entropic corrections). The reverse rate constants are calculated from the forward reaction and equilibrium constants 


$$
k_{r}=\frac{k_{f}}{K_{e q}}
$$

with the equilibrium constant dependent on the species free energies via the reaction free energy

$$
K_{e q} \propto \exp \left(\frac{-\Delta G_{r x n}}{R T}\right)
$$

The species free energies incorporate lateral interactions using the qualitative model

$$
G_{j}=G_{j 0}+\sum_{i} \omega_{i j} \theta_{i}
$$

where $\omega_{i j}$ is the energy (lateral interaction) by which species $i$ affects species $j, \theta_{i}$ is the surface coverage of species $i$, and $G_{j 0}$ is the zero-coverage species free energy. Adsorbate interactions can also strongly affect the stability of transition states and thus the activation energies.(Mhadeshwar et al., 2004; Park et al., 1999) We have accounted for this with a simple qualitative model. The influence of coverage effects on the reaction free energy can readily be calculated from the species energies

$$
\Delta G_{r x n}=\sum_{j} v_{j}\left(G_{j 0}+\sum_{i} \omega_{i j} \theta_{i}\right)=\Delta G_{r x n 0}+\sum_{j} v_{j} \sum_{i} \omega_{i j} \theta_{i}
$$

where $v_{j}$ is the stoichiometric coefficient of species $j$ in the ith reaction. The last term on the right hand side represents the change in the reaction free energy due to lateral interactions. Half this change is added to the zero coverage activation energy

$$
E_{A}=E_{A 0}+\frac{1}{2} \sum_{j} v_{j} \sum_{i} \omega_{i j} \theta_{i}
$$

to generate an estimate for the coverage-dependent activation energy. The interaction parameters in this work are estimated based on literature,(Christiansen and Vlachos, 2012; Salciccioli et al., 2011a; Salciccioli and Vlachos, 2011; Sutton et al., 2013) although in principle one could also use DFT for this purpose as described elsewhere.(Mhadeshwar et al., 2004) The relevant adsorbate interaction parameters are listed in Table 2, with the values used in the FPSEM model being a pure subset of those employed in the DFT model.

Table 2. Adsorbate interaction parameters ( $\mathrm{kcal} / \mathrm{mol} / \mathrm{ML})$ used in microkinetic models. Interactions for $\mathrm{CH}_{3} \mathrm{C}^{*}$ are only included in the DFT model.

\begin{tabular}{lrrrr} 
& \multicolumn{4}{c}{ Modifying Species } \\
Modified Species & $\mathrm{CO}^{*}$ & $\mathrm{H}^{*}$ & $\mathrm{CH}_{3} \mathrm{C}^{*}$ & $\mathrm{CH}^{*}$ \\
\hline $\mathrm{CO}^{*}$ & -15 & & -15 & -15 \\
$\mathrm{H}^{*}$ & & -3 & & \\
$\mathrm{CH}_{3} \mathrm{CO}^{*}$ & -10 & & & \\
$\mathrm{CH}_{3} \mathrm{C}^{*}$ & -15 & & -15 & -30 \\
$\mathrm{CH}^{*}$ & -15 & -15 & -30
\end{tabular}




\section{Microkinetic Modeling}

The microkinetic models were solved numerically using an in-house Fortran code incorporating the Chemkin-II and Surface Chemkin libraries.(Coltrin et al., 1991; Kee et al., 1991) For improved numerical reliability, we have reformulated our earlier algorithm(Sutton et al., 2013) to solve a steady state PFR as a series of transient CSTRs integrated to steady state rather than solving the PFR directly (the former gives one system of ODEs for each CSTR, whereas the latter generates a system of differential-algebraic equations that are often more challenging to converge). The results in this work are based on the revised algorithm and compare well with the results of our previous model.(Sutton et al., 2013)

To identify species enthalpies and reaction activation energies requiring refinement, we employ brute force local sensitivity analyses (LSA), whereby we perturb each parameter of interest one-at-a-time and recalculate the model response for the perturbed parameter set. We then calculate the normalized sensitivity (NSC),(Sutton et al., 2013) for the $i$-th parameter $A_{i}$ to be

$$
N S C_{i}=\frac{d \ln X}{d \ln A_{i}} \approx \frac{A_{i} \Delta X}{X \Delta A_{i}}
$$

and $\mathrm{X}$ is a response, such as the conversion, the mass fraction of a gaseous species, the surface coverage of a species, the selectivity, etc. In this formulation, the parameter $A_{i}$ is assumed to be

$$
A_{i}=\exp \left(\frac{-E_{i}}{R T}\right)
$$

where $E_{i}$ is the associated species or activation energy we perturb. This definition is analogous to the sensitivity metrics for species and transition state energies proposed by Campbell and coworkers(Stegelmann et al., 2009) and is used to ensure that the resulting NSC is on the same numerical scale as our previous NSCs calculated using reaction pre-exponentials as the independent parameters. (Sutton et al., 2013) The NSCs give insight into which parameters are the most influential both for promoting and inhibiting the model responses.

\section{Hierarchical Refinement Algorithm}

The algorithmic approach to hierarchical refinement advocated in this work is represented in Figure 2. The algorithm consists of two basic loops: an inner loop and an outer loop. The outer loop involves three steps: (1) postulating the model, (2) refining the postulated model (the inner loop), and (3) comparing to experimental data including design of experiments for model assessment. If the model describes adequately the data (Step 3), further refinement is not pursued. Otherwise, the mechanism requires additional chemical or physical insights, and the process begins again with an updated mechanism (e.g., adding reaction paths, exploring a different crystallographic plane, considering support effects, etc.).

Although a complete discussion of the outer loop is beyond the scope of this work, a basic understanding of the associated concepts is vital for successfully employing the hierarchical refinement methodology. As an example, we refer to our previously published model of ethanol steam reforming on 
$\mathrm{Pt} / \mathrm{Al}_{2} \mathrm{O}_{3}$. (Sutton et al., 2013) When developing that model, we proposed that the mechanism consists of the elementary reactions comprising thermal decomposition and water-gas shift mechanisms. These elementary reactions were proposed based on prior work in the literature (primarily published DFT calculations) and were assumed to occur exclusively on the close-packed (111) facet of Pt. We then performed our own DFT calculations to parameterize the model and numerically solved the resulting microkinetic model. Upon comparison to experimental data, we determined that while the model gave quantitative results for the thermal decomposition portion of the mechanism, the water-gas shift mechanism required further tuning. For the purposes of that paper, key water-gas shift parameters were empirically adjusted to capture the qualitative trends. Future improvements to improve the performance of the water-gas shift mechanism might include incorporating step and edge sites and accounting for support effects.

The inner loop has four steps: (1) estimate the model parameters, (2) solve the parameterized model, (3) check model assumptions for self-consistency, and (4) identify the sensitive parameters. In Step 3 of the inner loop, we generally assess whether the model changes qualitatively from one iteration to the next. For example, a change in the dominant surface coverages requires adding lateral interactions when a species surface coverage surpasses a certain threshold (typically around 5-10\%). Similarly, a change in the most influential reactions requires refinement of the parameters of those reactions. Step 4 can be carried out in various ways, such as reaction path and sensitivity analyses (the method employed in this work).

In this context, a primary innovation of this work is in developing a systematic hierarchical classification scheme for parameter estimation methods used in Step 1 ranging from the inexpensive but less accurate FPSEM to the more expensive and more accurate ZPE-corrected DFT. Hierarchical refinement can be thought of as analogous to a Taylor expansion where at each iteration, one adds higher order terms, one at a time, to improve accuracy as the need is identified. With this analogy in mind, one can think of commonly employed methods as higher order accuracy terms. We summarize these in Table 3. 


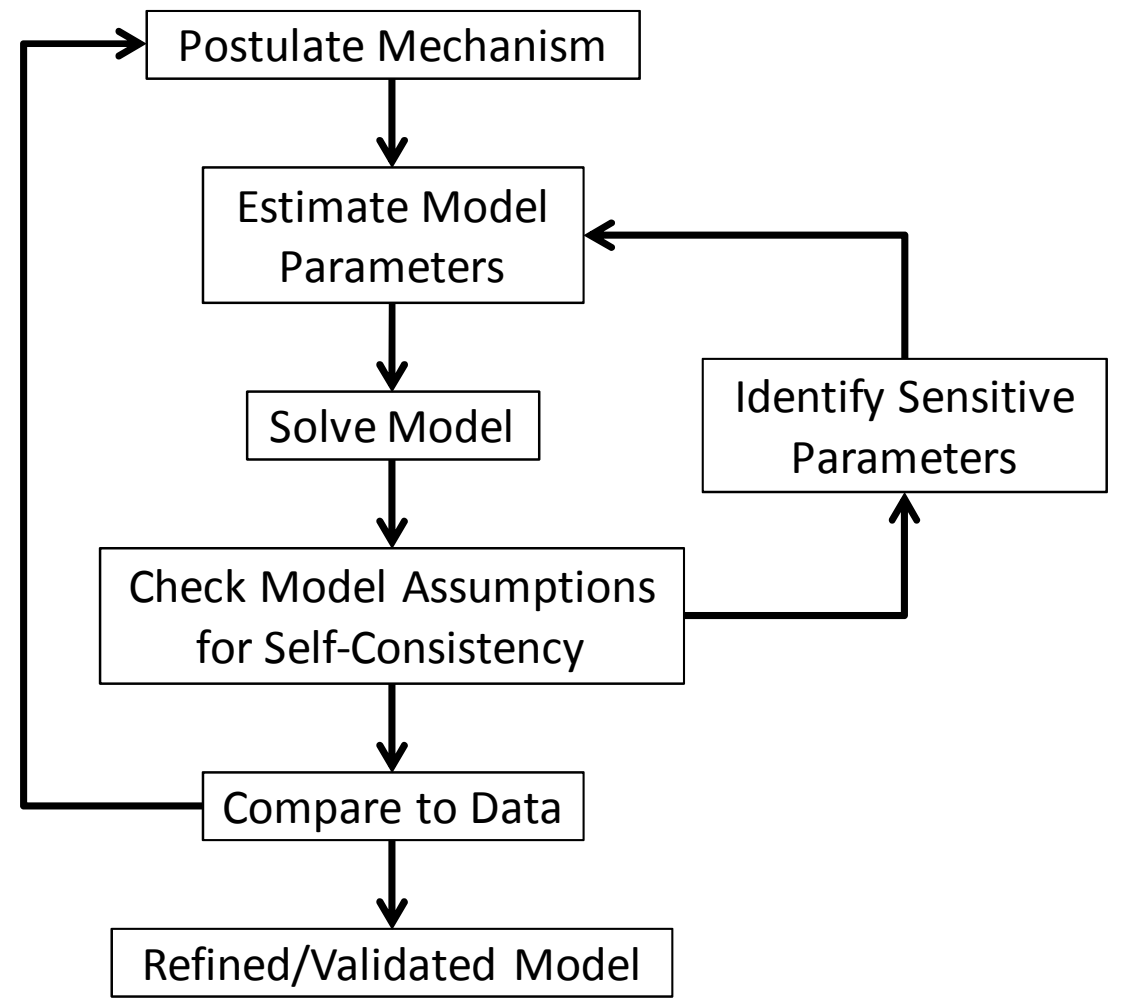

Figure 2. Schematic of hierarchical refinement algorithm.

Table 3. Summary of relative accuracy and typical computational times for popular parameter estimation methods.

\begin{tabular}{llll}
\multicolumn{1}{c}{ Method $^{1}$} & \multicolumn{1}{c}{ Parameter $^{2}$} & \multicolumn{1}{c}{$\begin{array}{c}\text { Order of } \\
\text { Accuracy }^{3}\end{array}$} & \multicolumn{1}{c}{$\begin{array}{c}\text { Time per } \\
\text { Calculation }^{4}\end{array}$} \\
\hline $\mathrm{GA}^{5}$ & $\begin{array}{l}\text { Species Free Energy } \\
\text { LSR }^{5}\end{array}$ & 0 & $1 \mathrm{~s}$ \\
& $\begin{array}{l}\text { Species Enthalpy or } \\
\text { Binding Energy }\end{array}$ & 0 & $1 \mathrm{~s}$ \\
$\mathrm{BEP}^{5}$ & $\begin{array}{l}\text { Reaction Activation Energy } \\
\text { TST }\end{array}$ & 0 & $1 \mathrm{~s}$ \\
DFT, no ZPE & $\begin{array}{l}\text { Pre-exponential } \\
\text { Species Energy \& Reaction }\end{array}$ & $1{\text { or } 2^{6}}^{6}$ & $1 \mathrm{~s}$ \\
DFT, ZPE & $\begin{array}{l}\text { Activation Energy } \\
\text { Species Free Energy \& }\end{array}$ & 2 & $1-10$ days \\
& $\begin{array}{l}\text { Reaction Activation Free } \\
\text { Energy }\end{array}$ & &
\end{tabular}

1. GA - Group Additivity; LSR - Linear Scaling Relation; BEP - Brønsted-Evans-Polanyi Correlation; TST - Transition State Theory; DFT - Density Functional Theory; ZPE - Zero-Point Energy Correction.

2. Free energy methods also yield enthalpies and entropies.

3. Accuracy order is defined in terms of a hypothetical series expansion, with higher orders corresponding to more accurate methods.

4. Applies only to the stated method. Time for performing DFT calculations for developing FPSEM is excluded.

5. Requires prior DFT calculations to generate correlation(s) for specified parameter.

6. First (second) order in the absence (presence) of entropic contributions. 
The zero-order accurate methods are all semi-empirical correlations (typically regressed from a set of DFT training data): group additivity (GA)(Benson and Buss, 1958; Benson et al., 1969; Chen et al., 2011; Cohen and Benson, 1993; Kua et al., 2000; Salciccioli et al., 2012) for estimating the free energies of species from group values, the linear scaling relations (LSR)(Abild-Pedersen et al., 2007; Fernández et al., 2008; Jones et al., 2011; Salciccioli et al., 2010; Sutton and Vlachos, 2013) for transferring previously estimated thermochemistry from one metal to another, and the Brønsted-Evans-Polanyi (BEP) relations(Alcala et al., 2003; Bligaard et al., 2004; Ferrin et al., 2009; Michaelides et al., 2003; Pallassana and Neurock, 2000; Salciccioli et al., 2011b; Sutton and Vlachos, 2012; Van SANTEN et al., 2007) for estimating activation energies from reaction energies. These correlations are all linear, and thus, kinetic parameters are accessible in real time and thereby enable the construction of high throughput microkinetic models for catalyst screening.(Ferrin et al., 2009) The first-order techniques are characterized by lacking ZPE and finite temperature corrections, whereas the second order techniques apply these corrections. Under this classification, TST is first order for the traditional order of magnitude estimate $k_{B} T / h$ and second order when the entropic contributions are included. Similarly, 0 K DFT energies (whether species energies or activation energies) are classified as first order, and the temperature/ZPE-corrected equivalents are thought of as second order. Likewise, parameter estimation methods for coverage effects on species and activation energies range from the inexpensive order of magnitude estimates employed here to more elaborate techniques (e.g., cluster expansion Hamiltonians parameterized using large numbers of DFT calculations).

In general, we recommend zero-order FPSEM methods (GA, LSRs, and BEPs) for generation of an initial model. Then, as sensitive parameters are identified, higher order methods are applied. For important species energies, we recommend using ZPE-corrected DFT in order to ensure accurate thermodynamic predictions at all temperatures. For activation energies, we find that non-ZPE corrected DFT is often adequate, with only a few important activation energies benefiting from the additional accuracy afforded from ZPE correction. In this approach, ZPE corrections are applied to a select number of species and reaction barriers.

\section{Application of Hierarchical Refinement to Ethanol Steam Reforming}

To demonstrate the hierarchical refinement algorithm and quantify its effectiveness, we apply the methods to developing a microkinetic model of ethanol steam reforming. We then compare the refined FPSEM model to a version of our previously published DFT-based model,(Sutton et al., 2013) where the empirical adjustments to the key water-gas shift pre-exponentials intended to improve the $\mathrm{CO}_{2}$ selectivity have been excluded in order to focus on model refinement due to computational parameter refinement rather than via empirical fitting. For the purposes of this work, we consider the DFT-based model to be the exact solution.

\section{Convergence of FPSEM Model to Full Model during Refinement Process}

Our initial model did not include adsorbate lateral interactions under the assumption that these parameters are unknown for a new model. This initial model is termed Iteration 0 . Prior knowledge on adsorbate-adsorbate interactions is always good to include whenever available as this will improve the 
model predictions while accelerating model convergence. The parameters refined at each iteration are summarized in Table 4. The first two iterations did not employ local sensitivity analyses and resulted in the identification of the dominant surface species requiring lateral interactions. This preliminary set of iterations was considered converged when the dominant adsorbates (defined by a coverage of greater than $\sim 10 \%$ during at least one iteration) all included adsorbate interactions. The interaction parameters were obtained from order-of-magnitude estimates based on values for species in similar mechanisms.(Salciccioli et al., 2011a; Salciccioli and Vlachos, 2012) Subsequent iterations employed one-at-a-time brute force LSAs on the species enthalpies and reaction activation energies to identify the parameters requiring refinement. All LSA calculations were carried out at a single operating condition (specified in Table 4 and chosen as a representative operating condition from our previous work with this model). While in principle it is a best-practice to carry out LSA calculations at a variety of operating conditions (as the most influential species and reactions are dependent on operating condition), we found that this was not necessary for our particular system, as judged by the excellent agreement between the 'exact' DFT and approximate FPSEM models. In practice, such a determination would be made by comparison to experimental data.

The values of the NSCs determine parameters for refinement. For this system, the largest NSCs are of the order of 0.1. As such, we refine any parameters which have NSCs greater than 0.01 (absolute value). This threshold is one order of magnitude lower than the maximum and this is a rather conservative approach. An alternative is to refine only the highest one or two parameters at a time. Any GA-based sensitive species energy is automatically replaced with a DFT-derived free energy (this is owing to the importance of thermodynamics in ensuring a correct mechanism), whereas any BEP-derived activation energy is replaced initially with a $0 \mathrm{~K}$ activation energy upon first identification and with an activation free energy if a subsequent sensitivity analysis results in that parameter having an NSC larger than 0.1. Once a model parameter has been computed with the highest order technique deemed relevant, it is excluded from further consideration. Thus, very sensitive parameters, such as the activation energy for ethanol dehydrogenation to $\mathrm{CH}_{3} \mathrm{CHOH}^{*}$, were calculated with second-order techniques, whereas less sensitive parameters were estimated using only first-order techniques. Once the LSA identified no new parameters requiring refinement, the model refinement was terminated. Alternative stopping criteria are discussed below. 
Table 4. List of parameters refined at each iteration and their corresponding normalized sensitivity coefficients (NSCs). Operating conditions are $300^{\circ} \mathrm{C}, 1 \mathrm{~atm}, 12.5 \%$ ethanol, $37.5 \%$ water, bal. He in a PFR for identical catalyst surface areas.

\begin{tabular}{|c|c|c|}
\hline Iteration & Refined Parameters & NSC $^{1}$ \\
\hline 0 & Initial Model $^{2}$ & $\mathrm{~N} / \mathrm{A}$ \\
\hline 1 & $\mathrm{CH}-\mathrm{CH}$ Interaction Energy ${ }^{3}$ & $\mathrm{~N} / \mathrm{A}$ \\
\hline 2 & $\begin{array}{l}\text { CH Species Free Energy } \\
\text { CO-CO Interaction Energy }\end{array}$ & $\begin{array}{l}\text { N/A } \\
\text { N/A }\end{array}$ \\
\hline & $\mathrm{CO}-\mathrm{CH}$ Interaction Energy & $\mathrm{N} / \mathrm{A}$ \\
\hline & $\mathrm{CH}-\mathrm{CO}$ Interaction Energy & $\mathrm{N} / \mathrm{A}$ \\
\hline & $\mathrm{H}-\mathrm{H}$ Interaction Energy & $\mathrm{N} / \mathrm{A}$ \\
\hline 3 & $\mathrm{CH}_{3} \mathrm{CH}_{2} \mathrm{OH} \propto \mathrm{C}-\mathrm{H} 0 \mathrm{~K}$ Activation Energy ${ }^{5}$ & -0.50 \\
\hline & $\mathrm{CH}_{3} \mathrm{CHOH} \alpha \mathrm{C}-\mathrm{H} 0 \mathrm{~K}$ Activation Energy & 0.03 \\
\hline & $\mathrm{CH}_{3} \mathrm{C}-\mathrm{H} O \mathrm{~K}$ Activation Energy & -0.08 \\
\hline & $\mathrm{CH}_{3} \mathrm{CHOH} \mathrm{O}-\mathrm{H} O \mathrm{~K}$ Activation Energy & -0.04 \\
\hline & CHCO C-C 0 K Activation Energy & -0.11 \\
\hline & CCO C-C 0 K Activation Energy & -0.02 \\
\hline & $\mathrm{CH}_{3} \mathrm{CH}_{2} \mathrm{OH}$ Species Free Energy & -0.05 \\
\hline & $\mathrm{CH}_{3} \mathrm{CHOH}$ Species Free Energy & -0.47 \\
\hline & $\mathrm{CH}_{3} \mathrm{COH}$ Species Free Energy & 0.03 \\
\hline & $\mathrm{CH}_{3} \mathrm{CHO}$ Species Free Energy & -0.02 \\
\hline & $\mathrm{CH}_{3} \mathrm{CO}$ Species Free Energy & 0.01 \\
\hline & $\mathrm{CH}_{2} \mathrm{CO}$ Species Free Energy & 0.03 \\
\hline & CCO Species Free Energy & 0.08 \\
\hline & $\mathrm{CH}_{3}$ Species Free Energy & -0.01 \\
\hline 4 & $\begin{array}{l}\mathrm{CH}_{2} \text { Species Free Energy } \\
\mathrm{CHCO} \text { Species Free Energy }\end{array}$ & $\begin{array}{l}-0.07 \\
-0.06\end{array}$ \\
\hline & $\mathrm{CH}_{3} \mathrm{CH}_{2} \mathrm{OH} \propto \mathrm{C}-\mathrm{H}$ Activation Free Energy & -0.26 \\
\hline & $\mathrm{CH}_{3} \mathrm{CO} \beta \mathrm{C}-\mathrm{H} 0 \mathrm{~K}$ Activation Energy & -0.03 \\
\hline & $\mathrm{CH}_{2} \mathrm{CO} \beta \mathrm{C}-\mathrm{H} 0 \mathrm{~K}$ Activation Energy & -0.06 \\
\hline & $\mathrm{CHCO} \beta \mathrm{C}-\mathrm{H} O \mathrm{~K}$ Activation Energy & -0.06 \\
\hline & $\mathrm{CH}_{3} \mathrm{CO} \mathrm{C}-\mathrm{C} 0 \mathrm{~K}$ Activation Energy & -0.01 \\
\hline 5 & $\begin{array}{l}\mathrm{CH}_{3} \mathrm{CO}-\mathrm{CO} \text { Interaction Energy } \\
\mathrm{CHCO} \mathrm{C}-\mathrm{C} \text { Activation Free Energy }\end{array}$ & $\begin{array}{l}\mathrm{N} / \mathrm{A} \\
0.63\end{array}$ \\
\hline
\end{tabular}

1. NSC value is from a LSA on the previous iteration with conversion as the response.

2. Initial model is parameterized using group additivity for all species free energies and BrønstedEvans-Polanyi correlations for activation energies except for those species and reactions involved in a water-gas shift mechanism that were taken from the literature.(Stamatakis et al., 2011)

3. Species interaction energies were estimated using order of magnitude considerations.

4. Species and activation free energies include ZPE and temperature corrections. Activation energies at $0 \mathrm{~K}$ do not include the ZPE correction.

5. Reactions are written assuming the decomposition direction (e.g., $\mathrm{CH}_{3} \mathrm{CH}_{2} \mathrm{OH}^{*}$ gives $\mathrm{CH}_{3} \mathrm{CHOH}^{*}$ ). 
6. The corresponding NSC is for acetaldehyde selectivity.

Table 4 shows that the largest injection of refined parameters (with six $0 \mathrm{~K}$ DFT activation energies and nine species free energies) occurs in Iteration 3 as a result of sensitive parameters identified using a LSA on the Iteration 2 model that led to the addition of the most important lateral interactions $(\mathrm{CH}-\mathrm{CH}$, $\mathrm{CH}-\mathrm{CO}, \mathrm{CO}-\mathrm{CO}$, and $\mathrm{H}-\mathrm{H})$. Iteration 4 refines a single species energy $\left(\mathrm{CHCO}^{*}\right)$ and five activation energies, with one of them (the $\alpha \mathrm{C}-\mathrm{H}$ abstraction in ethanol to form $\mathrm{CH}_{3} \mathrm{CHOH}^{*}$ ) being a second-order correction (i.e., to include temperature effects). This iteration also implements a lateral interaction parameter (between $\mathrm{CH}_{3} \mathrm{CO}^{*}$ and $\mathrm{CO} *$ ). The final iteration (5) adds the temperature corrections to the $\mathrm{C}-\mathrm{C}$ cleavage of $\mathrm{CHCO}^{*}$ to make the model quantitative.

We find that the species energies are disproportionately represented in the refined parameters. Out of the 26 parameters refined (excluding two second-order corrections to activation energies), eleven of these are species energies (out of 53 surface species in the model), six are adsorbate interaction parameters (previously given in Table 2), and the remaining ten are activation energies (out of 160 reactions, note that the activation energies for the water-gas shift mechanism were from the literature, and many of the adsorption reactions were assumed to be non-activated). This suggests that, at least for ethanol steam reforming and likely other chemistries, correct thermodynamics (and coverage effects modifying the thermochemistry) are more important overall than the kinetics.

It is of interest to explore the impact of each iteration on the agreement between the refined FPSEM and DFT models. We do this by comparing the conversion, $\mathrm{C}$ selectivities for $\mathrm{CH}_{4}$ and $\mathrm{CO}$, and coverages for dominant adsorbates $\left(\mathrm{CH}_{3} \mathrm{CO}^{*}, \mathrm{CO}^{*}\right.$ and $\left.\mathrm{H}^{*}\right)$ between the 'exact' DFT-based model and the FPSEM model at various levels of refinement. These results are presented in Figure 3.

We find that the initial model, lacking all adsorbate interactions, is in extremely poor qualitative agreement with the DFT-based model. Interestingly, the introduction of the lateral interactions for the dominant adsorbates results in a model (Iterations 1 and 2) with much improved activity, selectivity, and coverage trends, indicating that the FPSEM methods are capable of producing a qualitatively reasonable model with only the additional cost of estimating adsorbate lateral interactions and DFT calculations for the thermochemistry of a few key (dominant) adsorbates. Note that all important adsorbate interactions are required for the qualitatively correct model; a single refinement (see Iteration 1 ) is not sufficient to achieve this. Injecting more DFT data (Iteration 3) results in further improvement in the conversion, the coverages of the dominant surface adsorbates (except for acetyl, $\mathrm{CH}_{3} \mathrm{CO}$, which is too abundant at low temperatures), and the selectivity to all products, but especially acetaldehyde. Introducing an additional set of refined parameters (Iteration 4 ) has mixed results. The conversion and coverages are nearly quantitative, but the $C_{2} / C_{1}$ branching ratio at low temperatures is seriously degraded. Performing one last refinement identified by a sensitivity analysis on the acetaldehyde selectivity- applying temperature corrections to $\mathrm{CHCO}^{*} \mathrm{C}-\mathrm{C}$ cracking as Iteration 5 - is sufficient to correct the $\mathrm{C}_{2} / \mathrm{C}_{1}$ branching ratio to result in a nearly quantitative model.

While it might be possible to refine the model further (e.g., to better predict selectivities to minor products), such an endeavor is not likely to be a profitable use of computational resources. For example, 
with the Iteration 5 model, a local sensitivity analysis on the ethane selectivity identifies six new species (all $C_{2}$ hydrocarbons) and fifteen new reactions (mostly hydrogenation and cracking of $C_{2}$ hydrocarbons) requiring refinement for the first round of refinement alone. This is a very large computational burden that is likely to have little, if any, impact on the quality of the model predictions. A larger threshold on the sensitivity coefficients would filter these parameters out. 

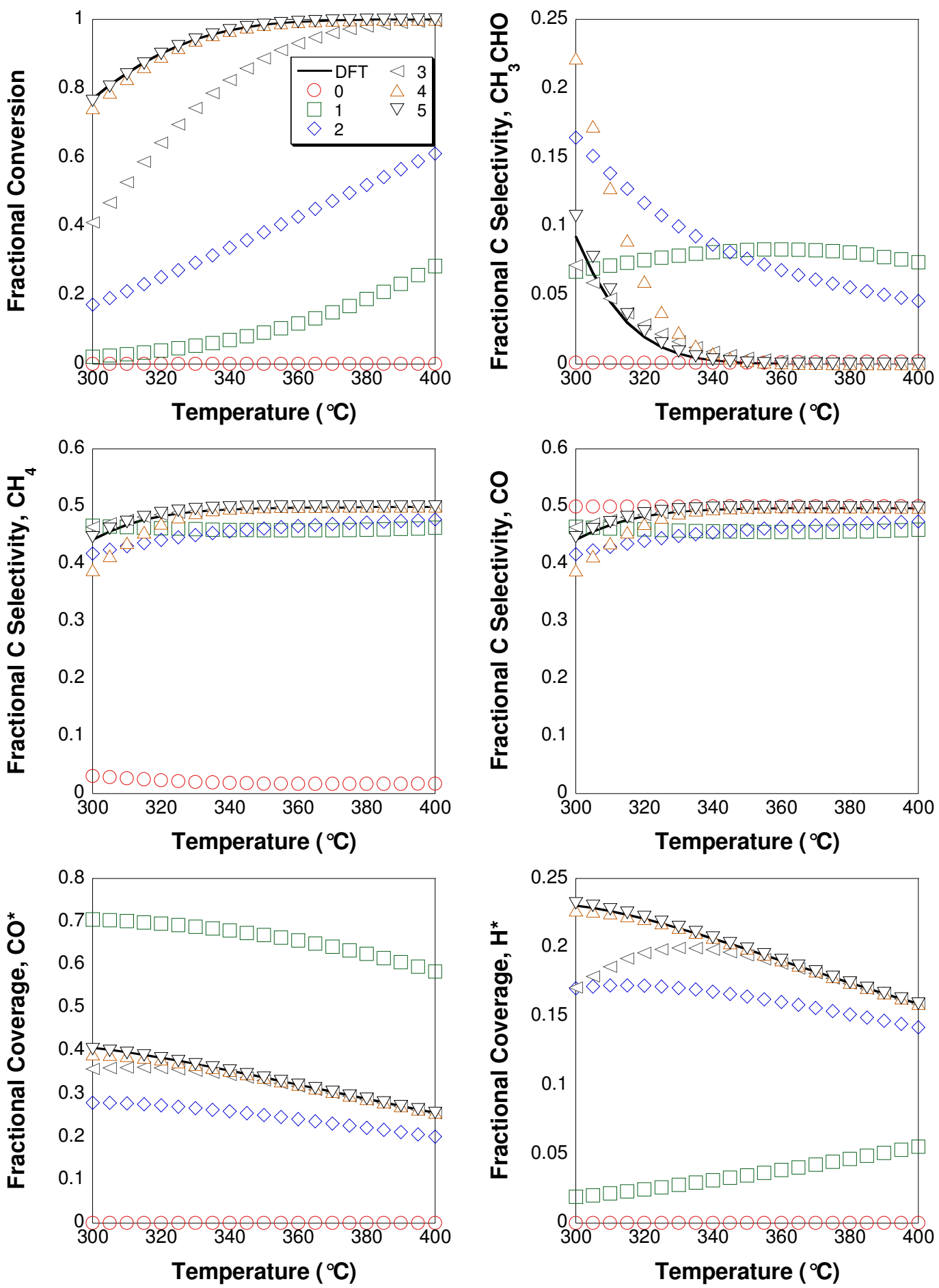

Figure 3. Convergence of fractional conversion, fractional $\mathrm{C}$ selectivity to major products $\left(\mathrm{CH}_{3} \mathrm{CHO}_{1} \mathrm{CH}_{4}\right.$, and $\left.\mathrm{CO}\right)$, and fractional coverages of major adsorbates (CO* and $\mathrm{H}^{*}$ ) for FPSEM-based model at selected iteration numbers (symbols) and DFT-based model (lines). At iteration 0 , the surface is almost completely poisoned by $\mathrm{CH}^{*}$ (coverage greater than 0.94 at all temperatures), and at Iteration $3, \mathrm{CH}_{3} \mathrm{CO} *$ is an important adsorbate (coverage of $18 \%$ at $300{ }^{\circ} \mathrm{C}$, dropping to less than $5 \%$ at $330{ }^{\circ} \mathrm{C}$ and to approximately zero at $400^{\circ} \mathrm{C}$ ). In other cases, the balance of the sites are vacant.

Another important aspect in understanding the model convergence is quantifying the speed with which the FPSEM model converges to the exact model. For this purpose, we define an error vector composed 
of the difference between the DFT- and FPSEM-based model mass fractions and coverages at all temperatures. We characterize how this error evolves over each iteration using three metrics: the Euclidean $\left(I_{2}\right)$ norm

$$
|\varepsilon|=\sqrt{\sum_{i} \varepsilon_{i}^{2}}
$$

where $\varepsilon_{i}$ are the elements of the error vector (i.e., the difference between individual mass fractions and coverages), the mean and standard deviation of all elements in the error vector, and the mean of the absolute values of the elements of the error vector. We summarize these results in Figure 4. Overall, we find that the total error as measured by all metrics, with the exception of the mean error, steadily decreases to near zero as the model is refined. Most of the error reduction occurs early in the refinement process and is due almost solely to the inclusion of adsorbate interactions, consistent with our earlier observation that the Iteration 2 model is qualitatively sound if quantitatively off. It predicts the correct rate determining step (ethanol dehydrogenation, see the NSC in Iteration 3, Table 4), the correct dominant products $\left(\mathrm{CH}_{4}\right.$ and $\mathrm{CO}$ as the primary products and $\mathrm{CH}_{3} \mathrm{CHO}$ as the main secondary product), and the correct dominant adsorbates ( $\mathrm{H}^{*}$ and $\left.\mathrm{CO}^{*}\right)$. Importantly, these early refinement steps are comparatively inexpensive relative to further refinements (we return to this point later). Additional refinements make the model quantitative but do not change the qualitative picture.
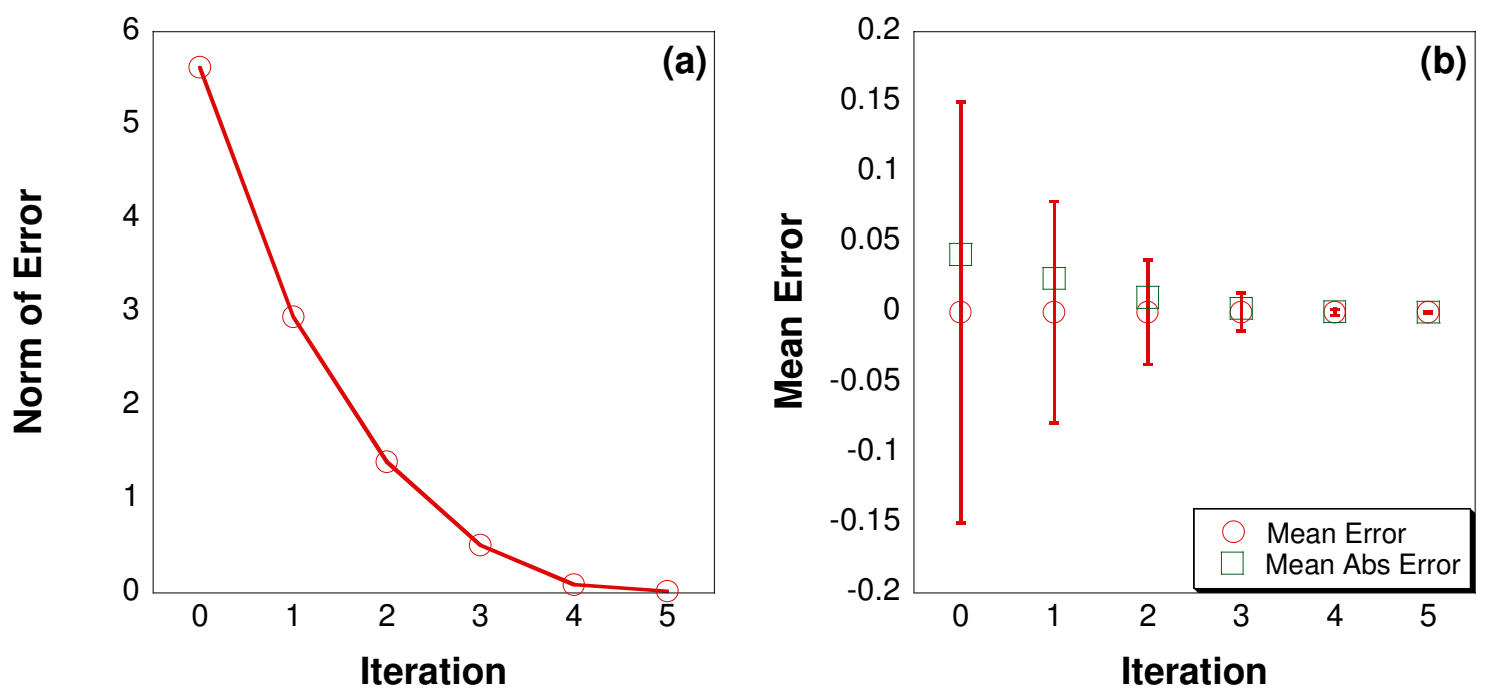

Figure 4. Error convergence rates for hierarchical refinement process. Panel (a) shows the Euclidean norm of the error vector. Panel (b) shows the mean and mean absolute errors. Error bars on the mean error in (b) denote one standard deviation of the error.

In the present example, we have terminated the refinement by monitoring the local NSCs on the conversion (all iterations) and selectivity to acetaldehyde (final iteration only) (see Table 4). At each iteration, if a new sensitive parameter is identified, the refinement continues. However, once the set of sensitive parameters is self-consistent (i.e., an LSA results in no new sensitive parameters), the refinement process is terminated. Other criteria or schemes might be chosen. For example, a relative change in the model solution could be used; if the model response changes by less than a certain tolerance, the refinement process would be terminated. Alternatively, the ultimate purpose of the 
model could also be taken into account. If only a qualitative model is needed (for example, as an exploratory or screening model or one which will ultimately be fit to data), a few iterations are likely all that is needed. On the other hand, if a model that captures the DFT model is needed, more refinement will necessarily be required.

\section{Comparison of Key Reaction Pathways for FPSEM and DFT Models}

In order to ensure that the refined model captures the correct reaction paths, we are interested in comparing the key reaction pathways in both the FPSEM-refined and DFT models by comparing the final reaction path analyses (Figure 5 ) and sensitivity analyses (
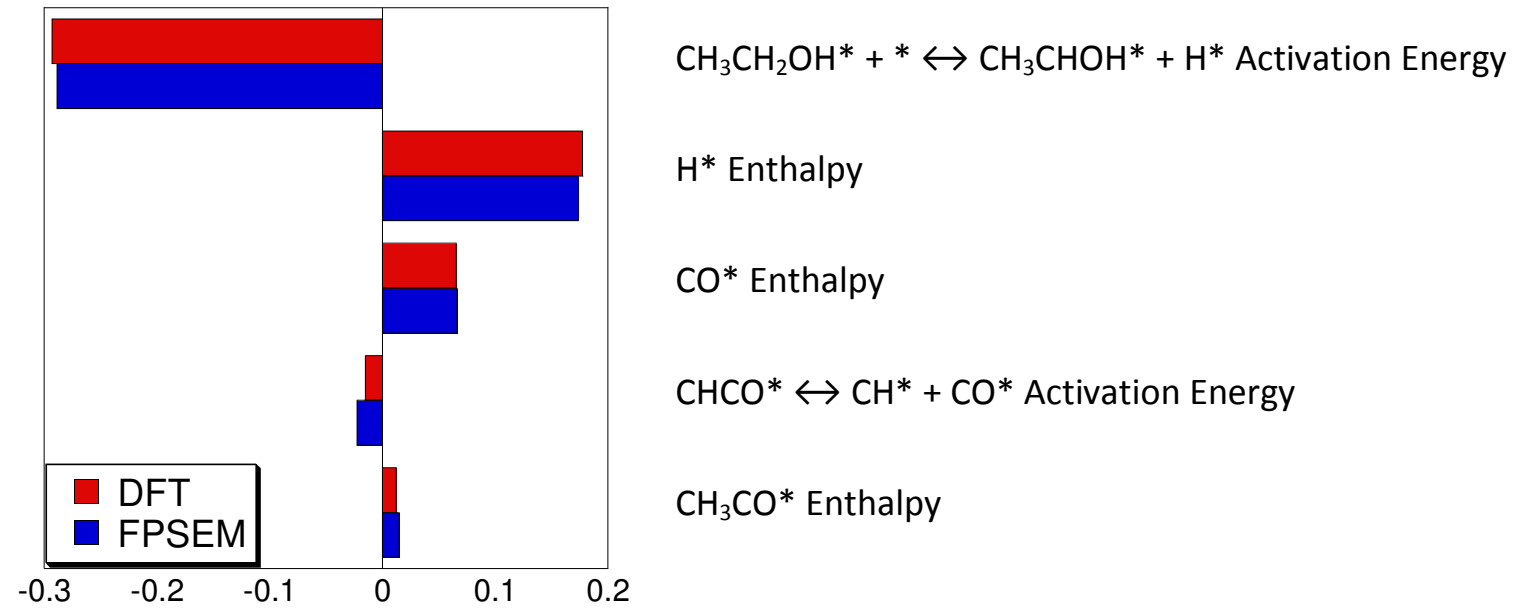

Normalized Sensitivity Coefficient

Figure 6). 


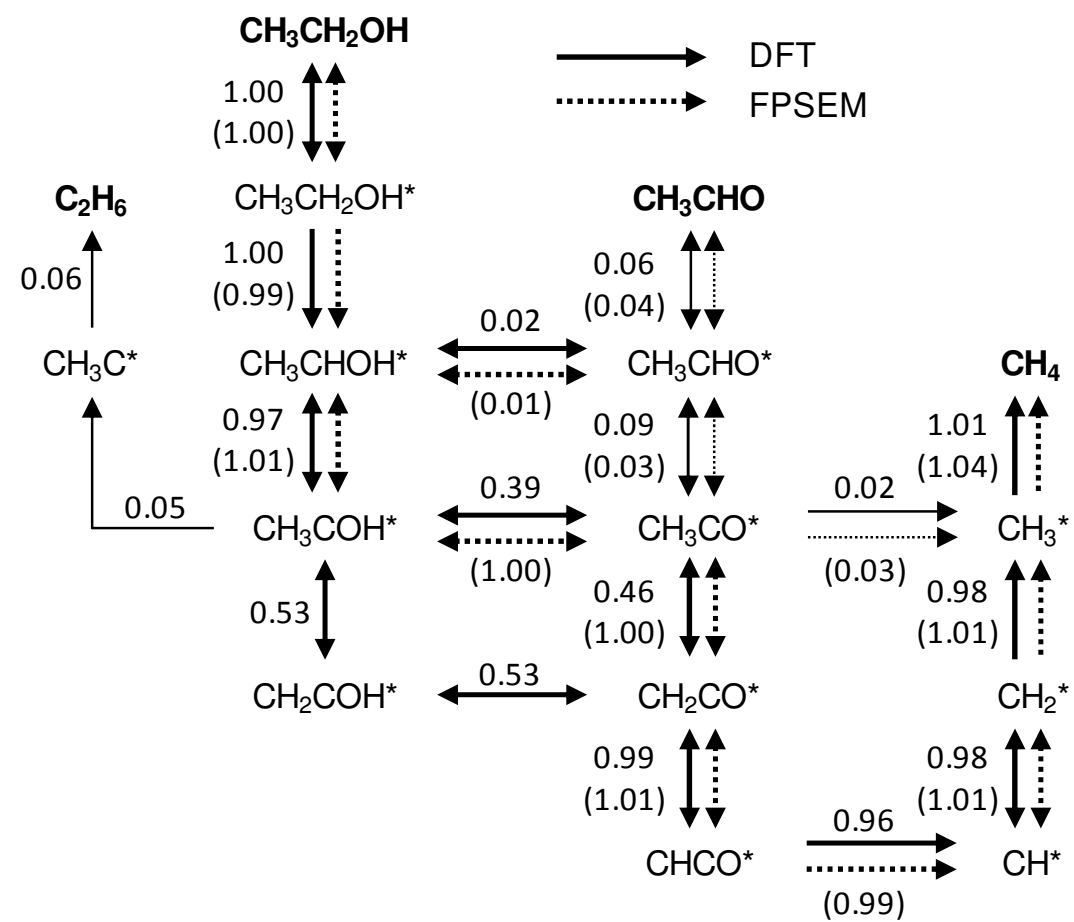

Figure 5. Reaction path analysis for DFT and refined FPSEM models at the reactor exit. For clarity auxiliary species and pathways (e.g., water-gas shift) have been omitted. Acetaldehyde $\left(\mathrm{CH}_{3} \mathrm{CHO}\right)$ acts as a secondary reactant, decomposing via acetyl $\left(\mathrm{CH}_{3} \mathrm{CO}^{*}\right)$ in both DFT and refined FPSEM models and $\mathrm{CH}_{3} \mathrm{CHOH}^{*}$ (refined FPSEM model only). Numbers are net reaction rates normalized by the net ethanol adsorption rate for the DFT (refined FPSEM in parentheses) models and reflect the rate in the decomposition direction (i.e., ethanol/acetaldehyde to methane). Single- (double-) headed arrows represent (ir)reversible reactions as determined from the partial equilibrium index (see our previous work(Sutton et al., 2013) for definition).

The two models are in excellent agreement with each other, as might be expected from our earlier results for the macroscopic responses (conversion, product selectivities, and surface coverages, Figure 3). In both models, the ethanol decomposition follows the path of dehydrogenation at the $\alpha \mathrm{C}$ to $\mathrm{CH}_{3} \mathrm{COH}^{*}$ followed by $\mathrm{O}-\mathrm{H}$ scission to $\mathrm{CH}_{3} \mathrm{CO}^{*}$. This species (acetyl) then dehydrogenates at the $\beta \mathrm{C}$ to CHCO* followed by $\mathrm{C}-\mathrm{C}$ cleavage. The $\mathrm{CH}_{x}$ species hydrogenate to methane, and some of the $\mathrm{CO}^{*}$ desorbs unreacted. The primary difference lies in the presence of some additional reactions that are active in the DFT-based model but inactive in the refined FPSEM model, most notably the production of ethane via ethylidyne $\left(\mathrm{CH}_{3} \mathrm{C}^{*}\right)$ produced as a result of the $\mathrm{C}-\mathrm{OH}$ cleavage of $\mathrm{CH}_{3} \mathrm{COH}^{*}$ and some additional equilibrated dehydrogenation reactions. 

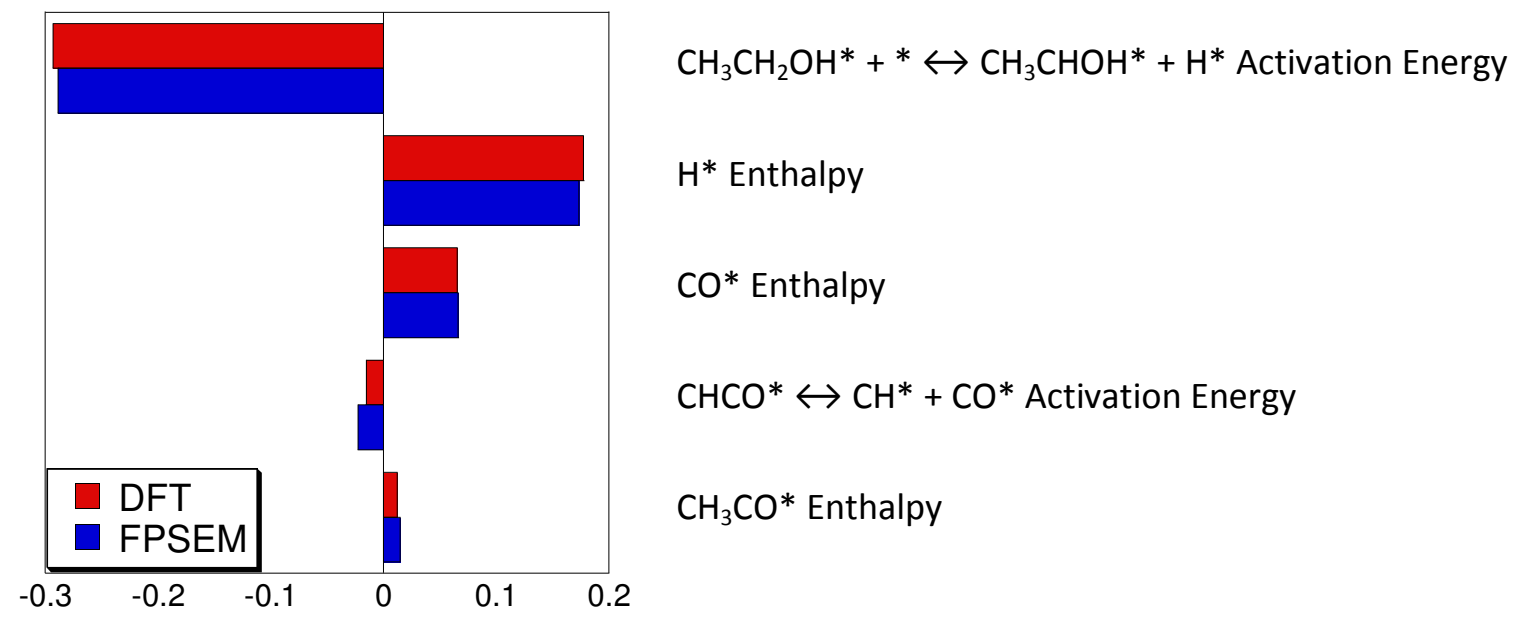

Normalized Sensitivity Coefficient

Figure 6. Normalized sensitivity coefficients at $300^{\circ} \mathrm{C}$ for DFT and final FPSEM refined microkinetic models for species enthalpies and reaction activation energies.

The normalized sensitivity coefficients (Figure 6

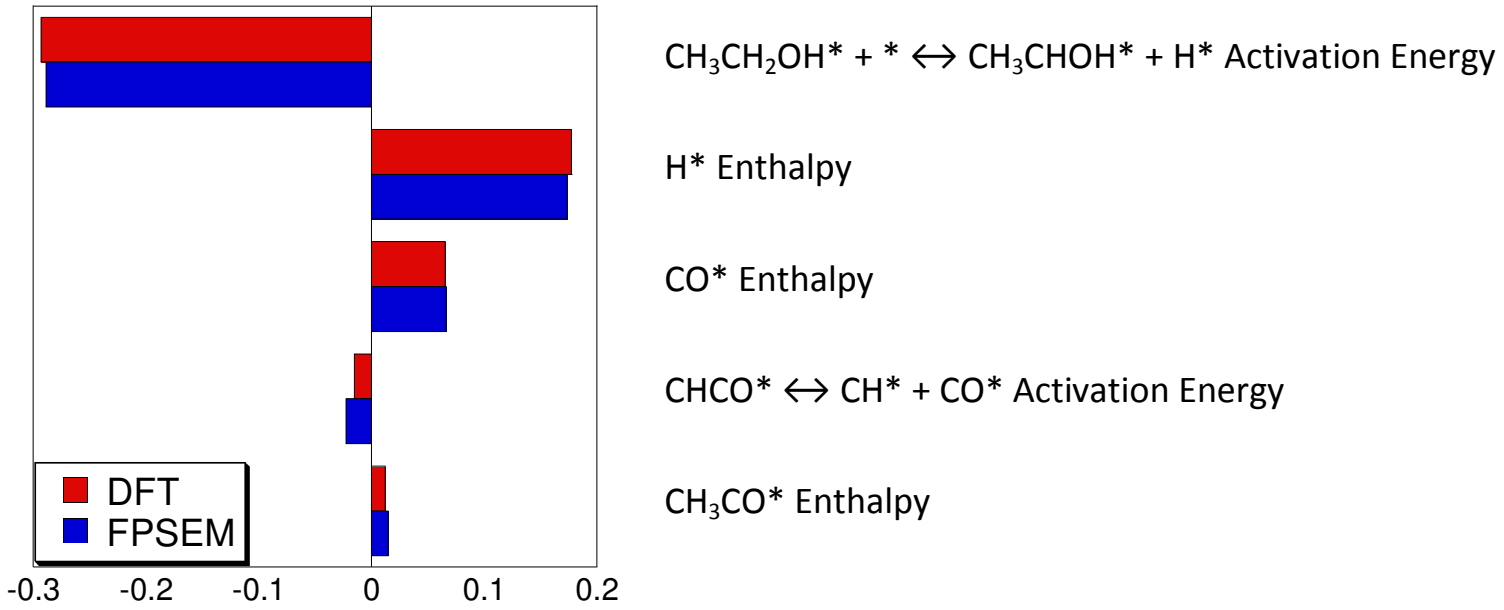

\section{Normalized Sensitivity Coefficient}

Figure 6) are in excellent quantitative agreement and indicate that the rate determining step is the initial dehydrogenation in ethanol. The other sensitive parameters are the enthalpies of $\mathrm{H}^{*}$ and $\mathrm{CO}^{*}$. While the sign of the NSC for the activation energy in ethanol is negative (implying a larger activation energy decreases the conversion), the signs for the $\mathrm{H}^{*}$ and $\mathrm{CO}^{*}$ enthalpies are positive. This indicates that more positive enthalpies (i.e., lowered adsorbate stability) in $\mathrm{H}^{*}$ and $\mathrm{CO}^{*}$ result in higher conversions. The increased desorption rates of these products, arising from their decreased surface stability, serves to drive the reversible upstream reactions towards the products.

\section{Estimated Time Savings from Hierarchical Refinement}

Since one of the aims of the hierarchical refinement is to reduce the computational time, we estimate the time required to refine the microkinetic model. Table 1Error! Reference source not found. presents an estimate for the DFT model. Given the DFT-based parameters required for the refinement (Table 4), 
the cumulative computational time vs. iteration is shown in Figure 7, assuming that the semi-empirical methods have previously been parameterized.

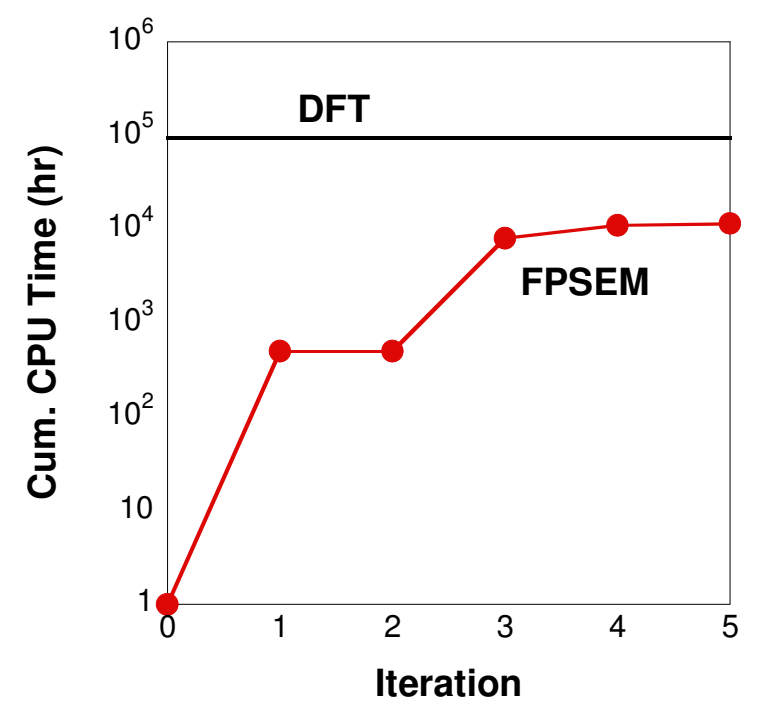

Figure 7. Breakdown of estimated cumulative computational time for DFT calculations for hierarchical refinement of the FPSEM-based model in CPU-hours as a function of iteration number. The total CPU time for the DFT-based model is shown for reference.

The initial model is nearly free (excluding the computational cost of parameterizing the FPSEM correlations), and the qualitative model (Iteration 2) requires, in addition to the order-of-magnitude interaction parameters (the DFT model uses the same values), a single DFT calculation (the free energy of $\mathrm{CH}^{*}$ ). The total computational time of the qualitative model is more than two orders of magnitude lower than that of the full model. The computational cost to make the model quantitative is at least an order of magnitude less than the exact model. Most of this cost is incurred in the first round of DFT calculations (Iteration 3), with subsequent refinement iterations requiring substantially fewer DFT calculations and adding minimally to the total time required to make the model quantitative.

If the semi-empirical methods have not been previously parameterized, then the computational savings will likely be reduced. A summary of estimated parameterization times for the semi-empirical methods is given in Table 5 for single molecule type for GA (e.g., a simple alcohol) or correlation/homologous series (LSR or BEP). If the model is moderately sized or smaller (e.g., ethanol steam reforming) such that complete DFT-parameterization is feasible, the cost to parameterize the FPSEM may represent a sizeable fraction of the completely DFT-based model and computational savings will be minimal. For larger models, the relative up-front cost is reduced, and the use of the FPSEM represents greater computational savings. Further, since the number of parameters needed for refinement is not expected to grow with model size, these savings will be even more profound for larger models that encompass many more parameters. The FPSEM are developed for an entire family of molecules and reactions and can be used at no cost to other members of each family. 
Table 5. Estimated computational times for parameterizing semi-empirical methods for each molecule type (GA) or correlation/homologous series (LSR, BEP).

\begin{tabular}{lrrr} 
FPSEM & CPU-hr/Calculation & Number of Calculations & Total CPU-hr \\
\hline GA & 500 & 30 & 15000 \\
LSR & 250 & 8 & 2000 \\
BEP & 500 & 8 & 4000
\end{tabular}

\section{Conclusions}

In this work, we have proposed a systematic hierarchical multiscale framework for building large microkinetic models with first principles' accuracy and reduced computational cost. The framework employs first-principles-based semi-empirical methods (FPSEM) to rapidly and inexpensively estimate initial parameter values and then systematically identifies influential parameters, via local sensitivity analysis, and refines them with DFT values according to a classification scheme, which is reminiscent of higher order corrections in an expansion. We then successfully applied this algorithm to develop qualitative and quantitative models of ethanol steam reforming using different stopping criteria. In doing so, we found that the FPSEMs are capable of constructing qualitatively correct microkinetic models with minimal refinement and greatly reduced computational cost (more than two orders of magnitude) compared to the full model. After sufficient refinement, quantitative models are also achievable, albeit for a smaller reduction in computational time (around one order of magnitude savings). These savings are expected to be much larger for bigger reaction mechanisms. Interestingly, species free energies and lateral interactions constitute the majority of refined parameters alluding to the importance of thermodynamics on the observed kinetic behavior.

\section{Acknowledgments}

We thank Dr. Michael Salciccioli for valuable discussions on local sensitivity analysis. This work was financially supported from the Catalysis Center for Energy Innovation, an Energy Frontier Research Center funded by the U.S. Department of Energy, Office of Science, Office of Basic Energy Sciences under Award Number DE-SC0001004.

\section{References}

Abild-Pedersen, F., Greeley, J., Studt, F., Rossmeisl, J., Munter, T.R., Moses, P.G., Skulason, E., Bligaard, T., Norskov, J.K., 2007. Scaling properties of adsorption energies for hydrogen-containing molecules on transition-metal surfaces. Phys Rev Lett 99, 016105.

Alcala, R., Mavrikakis, M., Dumesic, J., 2003. DFT studies for cleavage of C-C and C-O bonds in surface species derived from ethanol on Pt(111). Journal Of Catalysis 218, 178-190.

Benson, S.W., Buss, J.H., 1958. Additivity Rules for the Estimation of Molecular Properties Thermodynamic Properties. Journal Of Chemical Physics 29, 546-572.

Benson, S.W., Cruicksh.Fr, Golden, D.M., Haugen, G.R., Oneal, H.E., Rodgers, A.S., Shaw, R., Walsh, R., 1969. Additivity Rules for Estimation of Thermochemical Properties. CHEMICAL REVIEWS 69, 279-324. 
Blaylock, D.W., Ogura, T., Green, W.H., Beran, G.J.O., 2009. Computational Investigation of Thermochemistry and Kinetics of Steam Methane Reforming on Ni(111) under Realistic Conditions. Journal Of Physical Chemistry C 113, 4898-4908.

Bligaard, T., Norskov, J., Dahl, S., Matthiesen, J., Christensen, C., Sehested, J., 2004. The Bronsted-EvansPolanyi relation and the volcano curve in heterogeneous catalysis. Journal Of Catalysis 224, 206-217. Chen, Y., Salciccioli, M., Vlachos, D.G., 2011. An Efficient Reaction Pathway Search Method Applied to the Decomposition of Glycerol on Platinum. Journal Of Physical Chemistry C 115, 18707-18720. Chen, Y., Vlachos, D., 2010. Hydrogenation of Ethylene and Dehydrogenation and Hydrogenolysis of Ethane on Pt(111) and Pt(211): A Density Functional Theory Study. Journal Of Physical Chemistry C 114, 4973-4982.

Chen, Y., Vlachos, D.G., 2012. Density Functional Theory Study of Methane Oxidation and Reforming on Pt(111) and Pt(211). INDUSTRIAL \& ENGINEERING CHEMISTRY RESEARCH 51, 12244-12252.

Christiansen, M.A., Vlachos, D.G., 2012. Microkinetic modeling of Pt-catalyzed ethylene glycol steam reforming. Applied Catalysis A: General 431-432, 18-24.

Cohen, N., Benson, S.W., 1993. ESTIMATION OF HEATS OF FORMATION OF ORGANIC-COMPOUNDS BY ADDITIVITY METHODS. CHEMICAL REVIEWS 93, 2419-2438.

Coltrin, M.E., Kee, R.J., Rupley, F.M., 1991. Surface Chemkin (version 4.0): A FORTRAN package for analyzing heterogeneous chemical kinetics at a solid-surface-gas phase interface. Sandia National Laboratories, Livermore, CA.

Dumesic, J.A., Rudd, D.F., Aparicio, L.M., Rekoske, J.E., Trevino, A.A., 1993. The Microkinetics of Heterogeneous Catalysis. American Chemical Society, Washington, D.C.

Fernández, E.M., Moses, P.G., Toftelund, A., Hansen, H.A., Martínez, J.I., Abild-Pedersen, F., Kleis, J., Hinnemann, B., Rossmeisl, J., Bligaard, T., Nørskov, J.K., 2008. Scaling relationships for adsorption energies on transition metal oxide, sulfide, and nitride surfaces. Angew. Chem. Int. Ed. Engl. 47, 46834686.

Ferrin, P., Simonetti, D., Kandoi, S., Kunkes, E., Dumesic, J., Norskov, J., Mavrikakis, M., 2009. Modeling Ethanol Decomposition on Transition Metals: A Combined Application of Scaling and Bronsted-EvansPolanyi Relations. Journal Of The American Chemical Society 131, 5809-5815.

Goos, E., Burcat, A., Ruscic, B., 2014. Extended Third Millenium Ideal Gas Thermochemical Database with Updates from Active Thermochemical Tables.

Jones, G., Studt, F., Abild-Pedersen, F., Norskov, J.K., Bligaard, T., 2011. Scaling relationships for adsorption energies of C-2 hydrocarbons on transition metal surfaces. Chemical Engineering Science 66, 6318-6323.

Kee, R.J., Rupley, F.M., Miller, J.A., 1991. Chemkin-II: A FORTRAN chemical kinetics package for the analysis of gas phase chemical kinetics. Sandia National Laboratories, Livermore, CA.

Kua, J., Faglioni, F., Goddard, W., 2000. Thermochemistry for hydrocarbon intermediates chemisorbed on metal surfaces: $\mathrm{CHn}-\mathrm{m}(\mathrm{CH} 3)(\mathrm{m})$ with $\mathrm{n}=1,2,3$ and $\mathrm{m}<=\mathrm{n}$ on Pt, Ir, Os, Pd, Ph, and Ru. Journal Of The American Chemical Society 122, 2309-2321.

Mhadeshwar, A., Kitchin, J., Barteau, M., Vlachos, D., 2004. The role of adsorbate-adsorbate interactions in the rate controlling step and the most abundant reaction intermediate of NH3 decomposition on Ru. Catalysis Letters 96, 13-22.

Mhadeshwar, A., Vlachos, D., 2005. Hierarchical, multiscale surface reaction mechanism development: $\mathrm{CO}$ and $\mathrm{H}-2$ oxidation, water-gas shift, and preferential oxidation of $\mathrm{CO}$ on Rh. Journal Of Catalysis 234 , 48-63.

Michaelides, A., Liu, Z., Zhang, C., Alavi, A., King, D., Hu, P., 2003. Identification of general linear relationships between activation energies and enthalpy changes for dissociation reactions at surfaces. Journal Of The American Chemical Society 125, 3704-3705. 
Pallassana, V., Neurock, M., 2000. Electronic factors governing ethylene hydrogenation and dehydrogenation activity of pseudomorphic Pd-ML/Re(0001), Pd-ML/Ru(0001), Pd(111), and Pd$\mathrm{ML/Au(111)} \mathrm{surfaces.} \mathrm{Journal} \mathrm{Of} \mathrm{Catalysis} \mathrm{191,} \mathrm{301-317.}$

Park, Y.K., Aghalayam, P., Vlachos, D.G., 1999. A Generalized Approach for Predicting CoverageDependent Reaction Parameters of Complex Surface Reactions: Application to H2 Oxidation over Platinum. The Journal of Physical Chemistry A 103, 8101-8107.

Salciccioli, M., Chen, Y., Vlachos, D., 2010. Density Functional Theory-Derived Group Additivity and Linear Scaling Methods for Prediction of Oxygenate Stability on Metal Catalysts: Adsorption of OpenRing Alcohol and Polyol Dehydrogenation Intermediates on Pt-Based Metals. Journal Of Physical Chemistry C 114, 20155-20166.

Salciccioli, M., Chen, Y., Vlachos, D.G., 2011a. Microkinetic Modeling and Reduced Rate Expressions of Ethylene Hydrogenation and Ethane Hydrogenolysis on Platinum. INDUSTRIAL \& ENGINEERING CHEMISTRY RESEARCH 50, 28-40.

Salciccioli, M., Edie, S.M., Vlachos, D.G., 2012. Adsorption of Acid, Ester, and Ether Functional Groups on Pt: Fast Prediction of Thermochemical Properties of Adsorbed Oxygenates via DFT-Based Group Additivity Methods. The Journal of Physical Chemistry C 116, 1873-1886.

Salciccioli, M., Stamatakis, M., Caratzoulas, S., Vlachos, D.G., 2011b. A review of multiscale modeling of metal-catalyzed reactions: Mechanism development for complexity and emergent behavior. Chemical Engineering Science 66, 4319-4355.

Salciccioli, M., Vlachos, D.G., 2011. Kinetic Modeling of Pt Catalyzed and Computation-Driven Catalyst Discovery for Ethylene Glycol Decomposition. Acs Catalysis 1, 1246-1256.

Salciccioli, M., Vlachos, D.G., 2012. Kinetic Modeling of Pt-Catalyzed Glycolaldehyde Decomposition to Syngas. The Journal of Physical Chemistry A 116, 4621-4628.

Stamatakis, M., Chen, Y., Vlachos, D.G., 2011. First-Principles-Based Kinetic Monte Carlo Simulation of the Structure Sensitivity of the Water-Gas Shift Reaction on Platinum Surfaces. Journal Of Physical Chemistry C 115, 24750-24762.

Stegelmann, C., Andreasen, A., Campbell, C.T., 2009. Degree of Rate Control: How Much the Energies of Intermediates and Transition States Control Rates. Journal Of The American Chemical Society 131, 80778082.

Sutton, J.E., Panagiotopoulou, P., Verykios, X.E., Vlachos, D.G., 2013. Combined DFT, Microkinetic, and Experimental Study of Ethanol Steam Reforming on Pt. The Journal of Physical Chemistry C 117, 46914706.

Sutton, J.E., Vlachos, D.G., 2012. A Theoretical and Computational Analysis of Linear Free Energy Relations for the Estimation of Activation Energies. Acs Catalysis 2, 1624-1634.

Sutton, J.E., Vlachos, D.G., 2013. Error estimates in semi-empirical estimation methods of surface reactions. Journal Of Catalysis 297, 202-216.

Thybaut, J.W., Marin, G.B., 2013. Single-Event MicroKinetics: Catalyst design for complex reaction networks. Journal Of Catalysis 308, 352-362.

Van SANTEN, R., OFFERMANS, W., MALEK, K., PIDKO, E., 2007. Computational modeling of catalytic reactivity. Molecular Simulation 33, 327-336. 